\title{
Synergistic Antitumor Effects of Combined Treatment with HSP90 Inhibitor and PI3K/mTOR Dual Inhibitor in Cisplatin-Resistant Human Bladder Cancer Cells
}

\author{
Hyung Joon Kim ${ }^{1,2}$, Mi Kyung Gong ${ }^{3,4}$, Cheol Yong Yoon ${ }^{4}$, Jaeku Kang ${ }^{5}$, Mijin Yun ${ }^{2,6}$, \\ Nam Hoon $\mathrm{Cho}^{2,7}$, Sun Young $\mathrm{Rha}^{2,8}$, and Young Deuk $\mathrm{Choi}^{2,4}$ \\ ${ }^{1}$ Department of Urology, Myunggok Medical Research Institute, Konyang University College of Medicine, Daejeon; \\ ${ }^{2}$ Department of Medicine, Graduate School of Yonsei University College of Medicine, Seoul; \\ ${ }^{3}$ School of Dentistry, Pusan National University, Yangsan; \\ ${ }^{4}$ Department of Urology, Urological Science Institute, Yonsei University College of Medicine, Seoul; \\ ${ }^{5}$ Department of Pharmacology, College of Medicine, Konyang University, Daejeon; \\ ${ }^{6}$ Department of Nuclear Medicine, Yonsei University College of Medicine, Seoul; \\ ${ }^{7}$ Department of Pathology, Yonsei University College of Medicine, Seoul; \\ ${ }^{8}$ Division of Medical Oncology, Department of Internal Medicine, Yonsei University College of Medicine, Seoul, Korea.
}

Purpose: The current study aimed to investigate the synergistic antitumor effect of combined treatment with 17-DMAG (HSP90 inhibitor) and NVP-BEZ235 (PI3K/mTOR dual inhibitor) on cisplatin-resistant human bladder cancer cells.

Materials and Methods: Human bladder cancer cells exhibiting cisplatin resistance (T24R2) were exposed to escalating doses of 17-DMAG (2.5-20 nM) with or without NVP-BEZ236 (0.5-4 $\mu \mathrm{M})$ in combination with cisplatin. Antitumor effects were assessed by CCK- 8 analysis. Based on the dose-response study, synergistic interactions between the two regimens were evaluated using clonogenic assay and combination index values. Flow cytometry and Western blot were conducted to analyze mechanisms of synergism.

Results: Dose- and time-dependent antitumor effects for 17-DMAG were observed in both cisplatin-sensitive (T24) and cisplatin-resistant cells (T24R2). The antitumor effect of NVP-BEZ235, however, was found to be self-limiting. The combination of 17DMAG and NVP-BEZ235 in a 1:200 fixed ratio showed a significant antitumor effect in cisplatin-resistant bladder cancer cells over a wide dose range, and clonogenic assay showed compatible results with synergy tests. Three-dimensional analysis revealed strong synergy between the two drugs with a synergy volume of $201.84 \mu \mathrm{M} / \mathrm{mL}^{2} \%$. The combination therapy resulted in G1-phase cell cycle arrest and caspase-dependent apoptosis confirmed by the Western blot.

Conclusion: HSP90 inhibitor monotherapy and in combination with the PI3K/mTOR survival pathway inhibitor NVP-BEZ235 shows a synergistic antitumor effect in cisplatin-resistant bladder cancers, eliciting cell cycle arrest at the G1 phase and induction of caspase-dependent apoptotic pathway.

Key Words: Bladder cancer, Cisplatin, NVP-BEZ235, 17-DMAG

\footnotetext{
Received: April 10, 2020 Revised: May 14, 2020

Accepted: May 21, 2020

Corresponding author: Young Deuk Choi, MD, PhD, Department of Urology, Urological Science Institute, Yonsei University College of Medicine, 50-1 Yonsei-ro, Seodaemun-gu, Seoul 03722, Korea.

Tel: 82-2-2228-2317, Fax: 82-2-312-2538, E-mail: youngd74@yuhs.ac

-The authors have no potential conflicts of interest to disclose.

(c) Copyright: Yonsei University College of Medicine 2020

This is an Open Access article distributed under the terms of the Creative Commons Attribution Non-Commercial License (https://creativecommons.org/licenses/ by-nc/4.0) which permits unrestricted non-commercial use, distribution, and reproduction in any medium, provided the original work is properly cited.
}

\section{INTRODUCTION}

One-third of patients with bladder cancer (BCa) exhibit muscle invasion at diagnosis, and half of them develop distant metastasis to the lungs, liver, and bone within 2 years. ${ }^{1}$ Cisplatinbased systemic chemotherapy is the first-line treatment option for metastatic BCa. Although the initial response rate for cisplatin chemotherapy is up to $50-70 \%$, most BCa patients experience recurrence, with a 5 -year survival rate for metastatic BCa of only $6 \% .^{2-4}$ In the last two decades, treatment options for 
these patients have not improved drastically. This may be due to drug resistance that often reduces the efficacy of cisplatin to cancer cells. At the same time, up to $50 \%$ of patients with $\mathrm{BCa}$ are ineligible for cisplatin, mostly because of decreased renal function. Over the past few decades, numerous trials have been conducted to overcome cisplatin resistance and to reduce the effective dose for preserving renal function.

Heat shock protein 90 (HSP90) is an intracellular protein that plays a crucial role as a chaperone. It acts in post-translational maturation and activation of many oncogenic client proteins. In healthy cells, the HSP90 chaperone complex is thought to work as a facilitator of cellular responses to extracellular signals. In cancer cells, HSP90 activates many oncogenic client proteins, stimulating cell survival, growth, and invasiveness. Therefore, HSP90 inhibitors are considered promising therapeutic options and are being investigated for use in several cancers preclinically. ${ }^{5-8}$ However, HSP90 inhibitor monotherapy has been found to show suboptimal inhibition of target proteins, ${ }^{9}$ and thus, combined approaches may be required..$^{10}$ Since some HSP90 targets have chemoprotective activity, the combination of an HSP90 inhibitor with a standard chemotherapeutic agent could markedly increase the in vivo efficacy of the therapeutic agent. ${ }^{11}$ As HSP90 acts on ERBB2 and its downstream signals (RAS-MEK-MAPK, PI3KAkt-mTOR), targeting both HSP90 and its downstream pathway could be potential strategy with which to overcome cisplatin resistance in human $\mathrm{BCa} .^{12,13}$

The PI3-Akt-mTOR signaling axis is known as a significant survival pathway. It is considered an essential target in cancer therapy since it plays a critical role in development, progression, metastasis, and chemoresistance. Several studies have shown a synergistic antitumor effect between PI3K or mammalian target of rapamycin (mTOR) inhibitors and conventional chemotherapies in chemo-naïve or resistant cancers, like melanoma, nasopharyngeal, and ovarian cancers. Unfortunately, PI3K or mTOR inhibitor monotherapy has not shown significant clinical outcomes as in HSP90 inhibitor monotherapy. In $\mathrm{BCa}$, however, simultaneous dual inhibition of PI3K/mTOR using NVP-BEZ235 has been found to exert a synergistic antitumor effect. ${ }^{3}$ Based on these results, we suspect to observe some synergism when both PI3K/mTOR dual inhibitor and HSP90 inhibitor are coupled in the treatment of cisplatin-resistant bladder cancer cells.

In the present study, we investigated the synergistic effects of combining the PI3K/mTOR pathway inhibitor NVP-BEZ235 and the HSP90 inhibitor 17-DMAG in cisplatin-based chemotherapy.

\section{MATERIALS AND METHODS}

\section{Cell lines and reagents}

Human bladder cancer cells (HTB9, J82, SW1710, T24, HTB5, UMUC14, and 253J) were maintained in MEM, DMEM, or
RPMI-1640, containing 10\% fetal bovine serum (GE Healthcare Hyclone, UT, USA), $100 \mathrm{U} / \mathrm{mL}$ penicillin, and $100 \mathrm{mg} / \mathrm{L}$ streptomycin (Gibco BRL, Grand Island, NY, USA). Cells were incubated at $37^{\circ} \mathrm{C}$ in a humidified $5 \% \mathrm{CO}_{2}$ atmosphere. UMUC14 cells were donated by Professor E.S. Lee (Seoul National University, Seoul, Korea), and all other cell lines were obtained from Korean Cell Line Bank (Seoul, Korea) or American Type Culture Collection (Manassas, VA, USA). The cisplatin-resistant T24R2 cell line was achieved by desensitizing T24 cells to cisplatin up to a concentration of $2 \mu \mathrm{g} / \mathrm{mL}$ (Fig. 1).

NVP-BEZ235 was provided by Novartis Pharmaceuticals Inc. (Basel, Switzerland), and 17-DMAG was purchased from Selleckchem (Selleckchem, Boston, MA, USA). NVP-BEZ235 compound was dissolved in $100 \% \mathrm{DMSO}$ at $85^{\circ} \mathrm{C}$ to prepare a $10 \mathrm{mM}$ stock solution and stored at $4^{\circ} \mathrm{C}$ until ready for use. Antibodies for p-AKT (ser473), AKT, p-mTOR (Ser2448), mTOR, p-GSK-3 $\beta$ (Ser9), GSK-3 $\beta$, p-4E-BP1 (Thr37/46), 4E-BP1, pMEK1/2 (Ser217/221), MEK1/2, p-ERK1/2 (Thr202/Tyr204), ERK1/2, cleaved Caspase (8 and 9), cleaved PARP, cIAP1, cIAP2, XIAP, LC3B, Beclin1, Cyclin A, Cyclin B1, Cyclin D1, and Cyclin E (Cell Signaling Technology, Danvers, MA, USA) and for Hsp90, ERBB2, and Bad (Santa Cruz Biotechnology, Santa Cruz, CA, USA) were used for immunoblotting.

\section{Dose-response and synergy study}

Bladder cancer cells were seeded on 96-well plates and were left to attach for 24 hours. For dose-response experiments, cells were treated with single and double combinations of NVP-BEZ235 (0.25-8.00 $\mu \mathrm{M})$ or 17-DMAG (0.98-1000 nM) for 24, 48, or 72 hours. Cytotoxic effects were evaluated by CCK-8 assay (Cell counting kit-8; Dojindo Molecular Technologies,

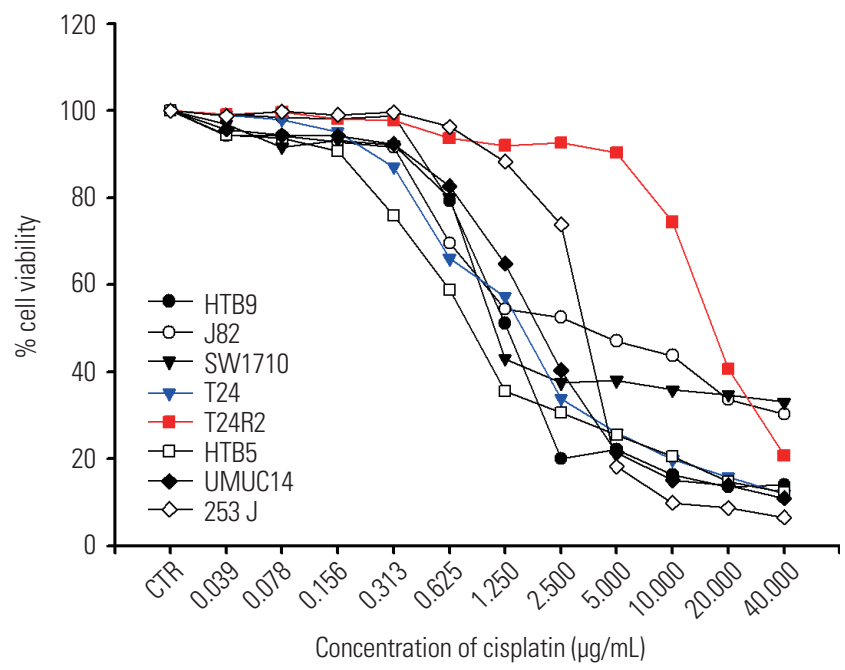

Fig. 1. Dose-response studies of cisplatin in human various bladder cancer cell lines. Human bladder cancer cells, including cisplatin-resistant T24R2, were exposed to increasing doses of cisplatin $(0.039-40.0 \mu \mathrm{g} / \mathrm{mL})$ for 48 hours, and changes in cell survival were assessed with the CCK-8 assay. Each data point represents a mean of at least three biological replicates. 
Gaithersburg, MD, USA) according to the manufacturer's protocol. Briefly, $10 \mu \mathrm{L}$ of CCK-8 reagent was added to each well at the end of the incubation period, and the plates were further incubated for 2 hours. Absorbance was read at an optical density of $450 \mathrm{~nm}$, and cell viability was expressed as the surviving fraction of cells in treated versus untreated controls.

The synergistic interaction between agents was quantitatively explored based on a combination index (CI) values using Calcusyn software (Biosoft, Ferguson, MO, USA) with the following interpretation: $\mathrm{CI}<1.0$ : synergism, $\mathrm{CI}>1.0$ : antagonism, and $\mathrm{CI}=1.0$ : additivity. The synergy between drugs was also assessed by a three-dimensional (3D) model using MacSynergy II software, in which areas of the graph above zero indicate synergism, while areas below zero indicate antagonism. The amount of synergy was expressed as surface area $(\mu \mathrm{M} /$ $\mathrm{mL}^{2} \%$ ), wherein $0-25,25-50,50-100$, and $>100 \mu \mathrm{M} / \mathrm{mL}^{2} \%$ indicated insignificant, weak, moderate, and strong interactions, respectively.

\section{Clonogenic assay}

For the colony-forming assay, T24R2 cells were seeded on a 6 -well plate at a concentration of $2 \times 10^{3}$ cells $/ \mathrm{mL}$ and treated with NVP-BEZ235 $(0.5 \mu \mathrm{M})$ and/or 17-DMAG $(2.5 \mathrm{nM})$ for 48 hours. Cells were cultured for another 2 weeks after removal of the drugs, and colonies formed were visualized by $0.4 \%$ crystal violet staining.

\section{Flow cytometric cell cycle analysis}

T24R2 cells were incubated with or without NVP-BEZ235 $(2 \mu \mathrm{M})$ or 17-DMAG (10 nM) for 48 hours, collected and fixed in $70 \%$ ethanol, and then, stained with a propidium iodide solution [970 $\mu \mathrm{L}$ PBS, $40 \mu \mathrm{L}$ of $1 \mathrm{mg} / \mathrm{mL}$ propidium iodide (Sig$\mathrm{ma}$ ), and $3 \mu \mathrm{L}$ of RNase A (Sigma)] for 30 minutes at $37^{\circ} \mathrm{C}$. After filtering with a strainer, the single-cell suspension was analyzed with a FACSCaliber flow cytometer (Becton-Dickinson, San Jose, CA, USA).

\section{Western blot analysis}

Cells were lysed using RIPA buffer supplemented with complete proteinase inhibitors (Roche, Basel, Switzerland). Cell lysates were then fractionated by SDS-PAGE and transferred to a PVDF membrane. Following blocking with $5 \%$ non-fat milk, the blots were incubated with corresponding primary antibodies and secondary antibodies. Signals were detected using a chemiluminescence Western blot substrate kit (Pierce, Rockford, IL, USA).

\section{Statistical analysis}

The data sets consisted of at least three biological replicates, and all statistical analyses were performed using SPSS $14.0 \mathrm{~K}$ software (SPSS Inc., Chicago, IL, USA). The data are expressed as a mean \pm SD. Statistical significance was determined by a two-sample t-test, and differences were considered significant at $p<0.05$.

\section{RESULTS}

\section{Synergistic antitumor effect between 17-DMAG and NVP-BEZ235}

Because HSP90 plays an import role as a chaperone in the activation and stabilization of ERBB2 and its downstream signals (RAS-MEK-MAPK, PI3K-Akt-mTOR), we hypothesized that concomitant targeting of HSP90 and its downstream path could be a promising strategy with which to overcome cisplatin resistance in human BCa (Fig. 2). Dual PI3K/mTOR inhibitor NVP-BEZ235 exerted dose- and time-dependent antitumor effects in both T24 and T24R2 cells (Fig. 3A and B). However, the antitumor effect of NVP-BEZ235 was found to be self-limiting, with all measures of its antitumor impact reaching a plateau at a concentration around $0.5 \mu \mathrm{M}$. When T24 and T24R2 cells were exposed to escalating doses of the HSP90 inhibitor 17DMAG, the proliferation of both cell lines was suppressed in a dose- and time-dependent manner (Fig. 3C and D). Interestingly, cisplatin-resistant T24R2 showed a relatively higher sensitivity to 17-DMAG treatment than the other human bladder cancer cell lines, including cisplatin-sensitive T24 (Fig. 4).

To investigate the synergistic interactions between two drugs, T24R2 cells were treated with escalating doses of 17DMAG alone or in a 1:200 fixed-ratio combination with NVPBEZ235, and the antitumor effects were evaluated by CCK-8 assay (Fig. 5A). The correlation coefficients (r) were between 0.792 and 0.982 , indicating fair data conforming to the median effect principle (Table 1). The Dms (IC50s) of NVP-BEZ235 and 17-DMAG were $2.08175 \mu \mathrm{M}$ and $0.01942 \mu \mathrm{M}$, respectively, while it was $0.00174 \mu \mathrm{M}$ in combination treatment, suggesting significant synergism between the two drugs. Fraction affected (fa) and $\mathrm{CI}$ analysis showed a $\mathrm{CI}<1$ over a wide range

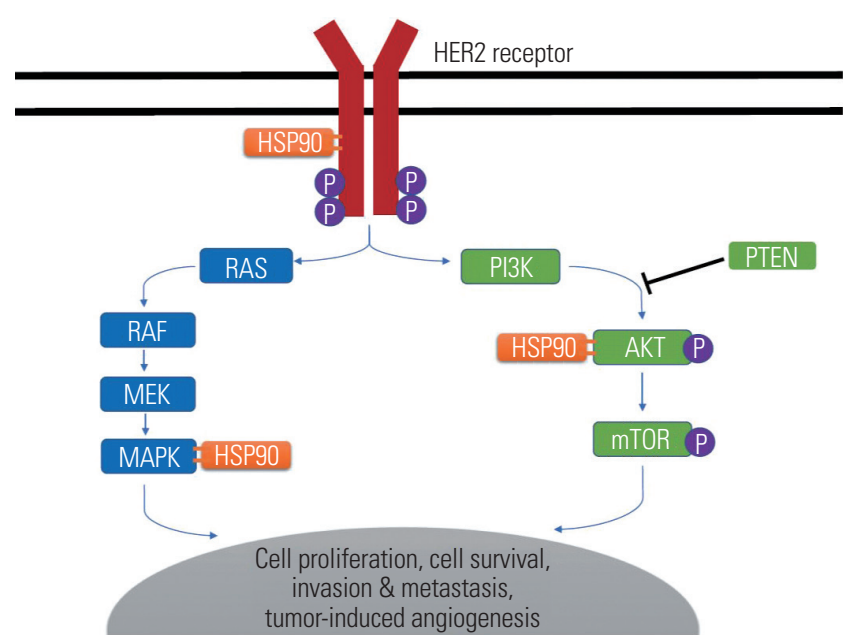

Fig. 2. A schematic diagram of crosstalk between HSP90 and PI3K-AKTmTOR/RAS-MEK-MAPK pathways. 

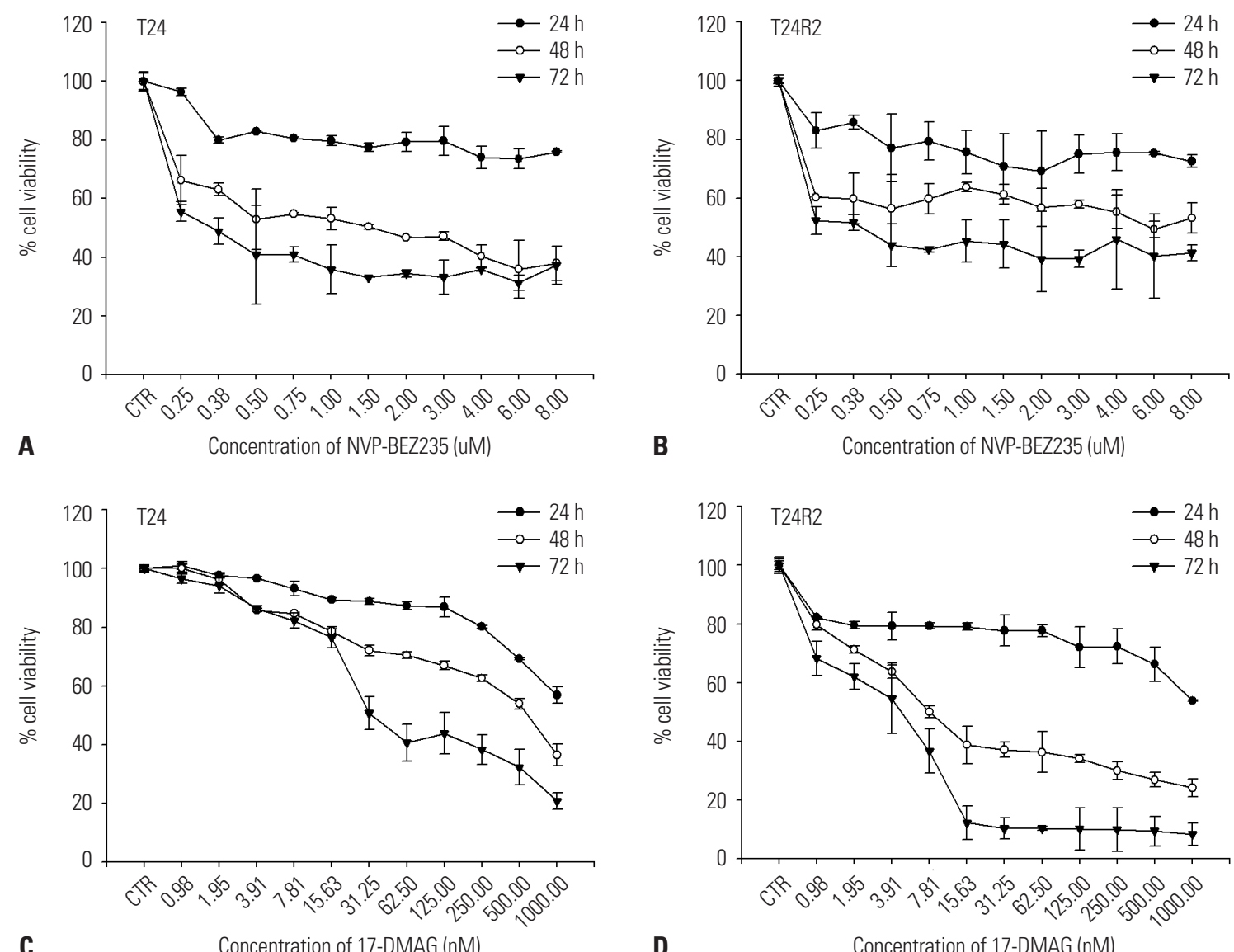

Fig. 3. Dose-response studies of 17-DMAG and NVP-BEZ235 in T24 and T24R2 cells. (A) Dose-response to NVP-BEZ235 in T24 cells. (B) Dose-response to NVP-BEZ235 in T24R2 cells. (C) Dose-response to 17-DMAG in T24 cells. (D) Dose-response to 17-DMAG in T24R2 cells. Both cell lines were exposed to escalating doses of 17-DMAG $(0.098 \mathrm{nM}-1 \mu \mathrm{M}$, upper panel) and NVP-BEZ235 (0.25-8.0 $\mu \mathrm{M}$, lower panel) for 24, 48, and 72 hours, and cell survival was assessed by the CCK-8 assay.

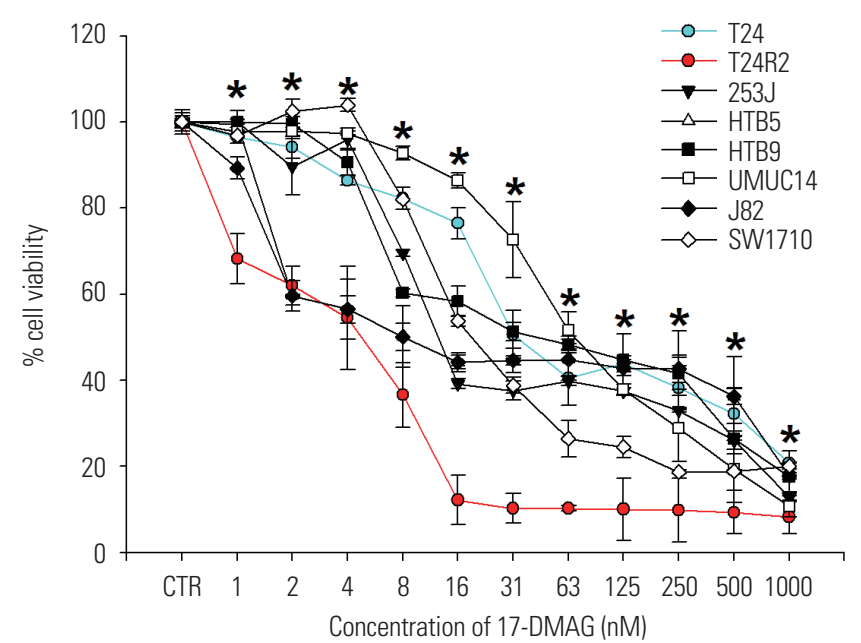

Fig. 4. Varying sensitivities of human bladder cancer cell lines to HSP90 inhibitor 17-DMAG. Human bladder cancer cell lines [T24 (grade 3), T24R2, 253J (grade 4), HTB5 (grade 4), HTB9 (grade 2), UMUC14 (grade 4), J82 (grade 3), and SW1710 (grade 3)] were treated with increasing doses of 17-DAMG $(1.0 \mathrm{nM}-1 \mu \mathrm{M})$ for 72 hours, and cell survival was evaluated with CCK-8 assay. ${ }^{*}$ Significant difference between T24 and T24R2 ( $p<0.05)$. of fractions affected (fa, 0.45-0.85). Clonogenic assay showed results compatible with the synergy test (Fig. 5E). For a more detailed analysis of synergism, four independent full combination studies with 17-DMAG and NVP-BEZ235 were performed to generate a 3D synergy plot using MacSynergy II software (Fig. 5D). The 3D analysis reflected strong synergy between the two drugs, with a synergy volume of $201.84 \mu \mathrm{M} / \mathrm{mL}^{2} \%$ and with minimal antagonism $\left(-10.18 \mu \mathrm{M} / \mathrm{mL}^{2} \%\right)$. The colonyforming assay results were compatible with the synergy test (Fig. 5E).

\section{Combined treatment with 17-DMAG and NVP-BEZ235 induces G1 and sub-G1 phase cell cycle arrest}

Treatment with neither 17-DMAG (10 nM) nor NVP-BEZ235 $(2.0 \mu \mathrm{M})$ alone elicited any significant alterations in cell cycle progression in T24R2 cells, whereas combined treatment resulted in marked increases in cells in the sub-G1 $(3.9 \pm 0.8 \%)$ and G1 $(78.3 \pm 0.7 \%)$ phases, compared with untreated control ( $0.6 \pm 0.3 \%$ for sub-G1, $57.9 \pm 1.3 \%$ for G1 phase), 17-DMAGtreated (1.4 $\pm 0.2 \%$ for sub-G1, $59.4 \pm 1.0 \%$ for G1 phase), and NVP-BEZ235-treated (1.0 $\pm 0.4 \%$ for sub-G1, $61.6 \pm 0.9 \%$ for G1 phase) cells (Fig. 6). 


\section{Synergy mechanisms between 17-DMAG and NVP-BEZ235}

Concomitant treatment of T24R2 cells with 17-DMAG and
NVP-BEZ235 suppressed cyclin A and B expression while causing increased expression of cyclin D (Fig. 7). Also, the combined treatment stimulated increased expression of caspase
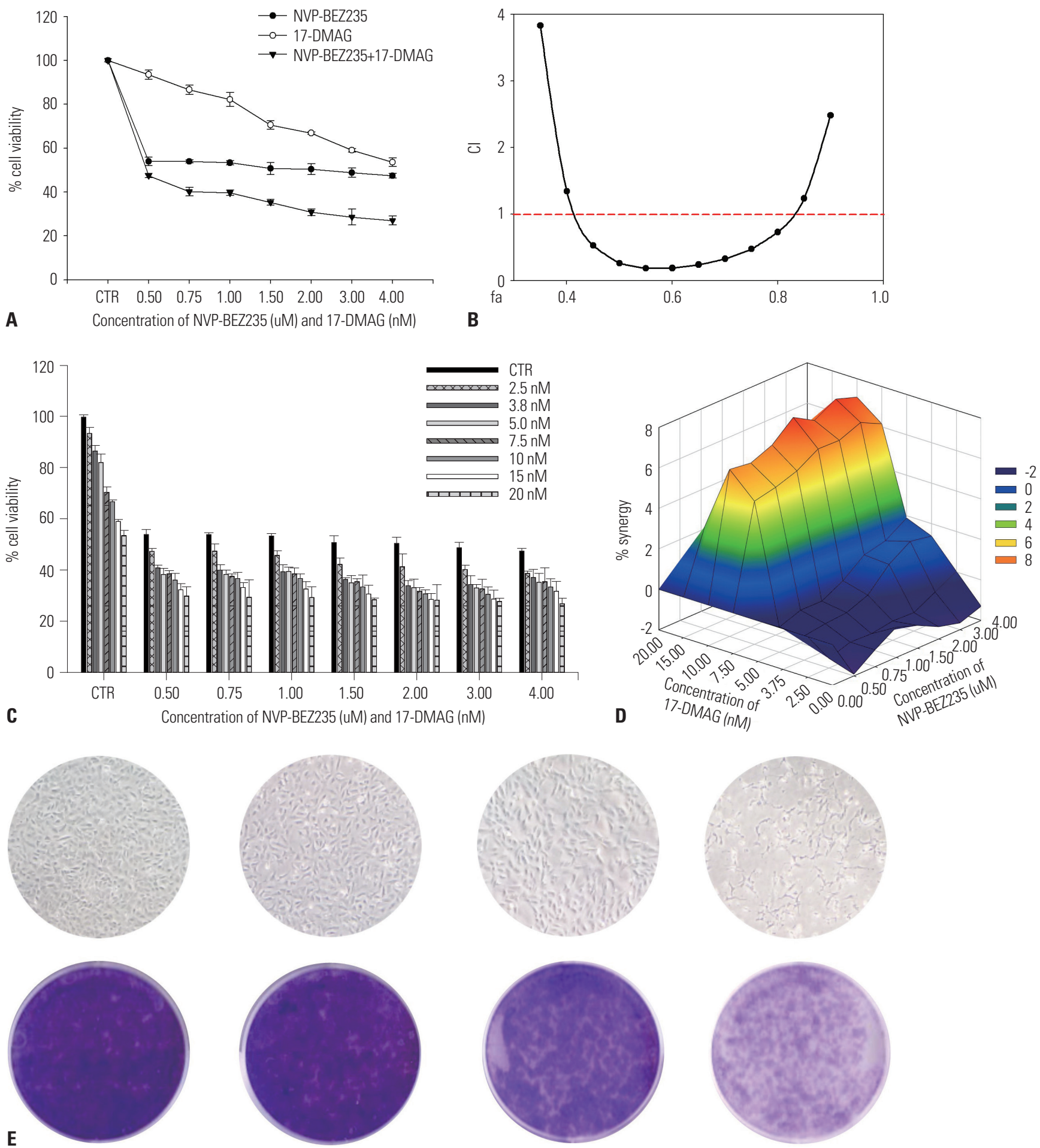

Fig. 5. Synergistic antitumor effects between 17-DMAG and NVP-BEZ235 in cisplatin-resistant T24R2 cells. (A) T24R2 cells were treated with escalating doses of 17-DMAG (2.5-20 nM) alone or in combination with NVP-BEZ235 $(0.5-4.00 \mu \mathrm{M})$ at a 1:200 fixed ratio for 72 hours. Cell survival was assessed using CCK-8 assay. (B) Based on the results of the CCK-8 assay, fa-Cl plots were generated in which fa and $\mathrm{Cl}$ stand for fraction affected and $\mathrm{Cl}$ values, respectively. (C) For more accurate test of synergism between the two drugs, T24R2 cells were treated with a full combination of 17-DMAG (2.5-20 nM) and NVP-BEZ235 $(0.5-4.00 \mu \mathrm{M})$ for 48 hours, and a 3D synergy plot was built using MacSynergy Il data analysis. (D) Clonogenic assay of T24R2 cells treated with 17-DMAG $(2.5 \mathrm{nM})$ alone or in combination with NVP-BEZ235 $(0.5 \mu \mathrm{M})$ for 48 hours. Cl, combination index; fa, fraction affected; 3D, three-dimensional. 
8/9 and PARP cleavage while suppressing the expression of cIAP1 and cIAP2 (Fig. 7). There were no discernible changes in beclin 1, LC3B1, and LC3B2 expression in T24R2 cells after combined treatment with 17-DMAG and NVP-BEZ235 (Fig. 7). Although there was no significant change in HSP90 expression by drug treatment, combined treatment suppressed ERBB2 and its downstream signals (p-Akt, Akt, p-mTOR, mTOR, p-4E-BP1, p-GSK-3 $\beta$ ) in T24R2 cells (Fig. 8).

\section{DISCUSSION}

Cisplatin-based chemotherapy is currently the gold standard for treating metastatic BCa. However, the survival benefit of the standard of care (SOC) has not seen improvement during the last few decades. A complete response is observed in only 1 out of 4 patients. Such a high treatment failure rate may partially be explained by the development of cisplatin resistance. Reduced cisplatin binding to DNA (pre-target resistance), reduced formation of DNA-cisplatin adducts (on-target resistance), altered DNA damage response (post-target resistance),

Table 1. Dose-Effect Relationships for 17-DMAG and NVP-BEZ235 in T24R2 Cells

\begin{tabular}{lccc}
\hline Compound & $\mathbf{m}^{*}$ & Dose $(\boldsymbol{\mu} \mathbf{M})^{\dagger}$ & $\mathbf{r}$ \\
\hline 17-DMAG & 1.185 & 0.01942 & 0.982 \\
NVP-BEZ235 & 0.135 & 2.08175 & 0.792 \\
Combination & 0.425 & 0.00174 & 0.988 \\
\hline
\end{tabular}

${ }^{*}$ Coefficient signifying dose-effect curve shape, ${ }^{\dagger} C_{50}$ or dose causing $50 \%$ inhibition of cell proliferation. and influenced signaling pathways that reduce the DNA damage response (off-target resistance) are believed to be viable mechanisms for cisplatin-resistance. ${ }^{14}$ Moreover, not all the patients are eligible for receiving a full therapeutic dose of cisplatin, mostly due to decreased renal function. Such profiles prompt alternative plans for sensitizing bladder cancer cells to chemotherapy and reducing the dose of cisplatin while enforcing its therapeutic efficacy. Due to a better understanding of tumor biology, sequencing after the SOC or combinations of novel agents are being recognized as promising options in treating malignancies, including genitourinary cancers.

There are several ongoing clinical trials of the treatment of advanced or metastatic urothelial carcinoma for both cisplatin-sensitive and -resistant cell lines. Various approaches, such as inhibitors of the mTOR, tyrosine kinase inhibitors, human epidermal growth factor receptor 2 (HER2) blockade, anti-angiogenic therapies, and immunotherapies, are being evaluated. Urothelial cancers are genetically complex with various oncogenic drivers and multiple mutations within a single tumor, gene fusion transcripts, copy number variations, and cytogenetic errors. Therefore, understanding the molecular mechanisms underlying $\mathrm{BCa}$ is important to developing effective treatments for cisplatin-resistant BCa. Interestingly, Lee, et al. ${ }^{14}$ revealed differential gene expression between T24 and T24R2 cells using microarray analysis: when T24 cells became resistant to cisplatin, various alterations were noted in the expression of genes controlling cell signaling, transcriptional regulation, metabolism, cytoskeleton, immunity, growth factor, transporter, adhesion, and apoptosis.

Recently, HER2 has emerged as a potent biomarker for many
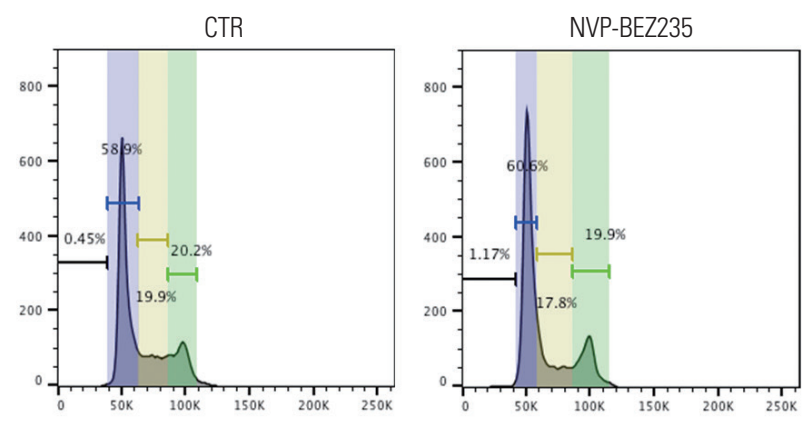

17-DMAG
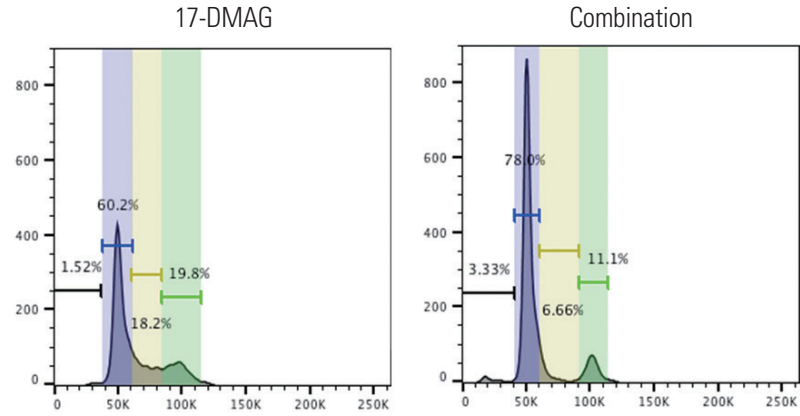
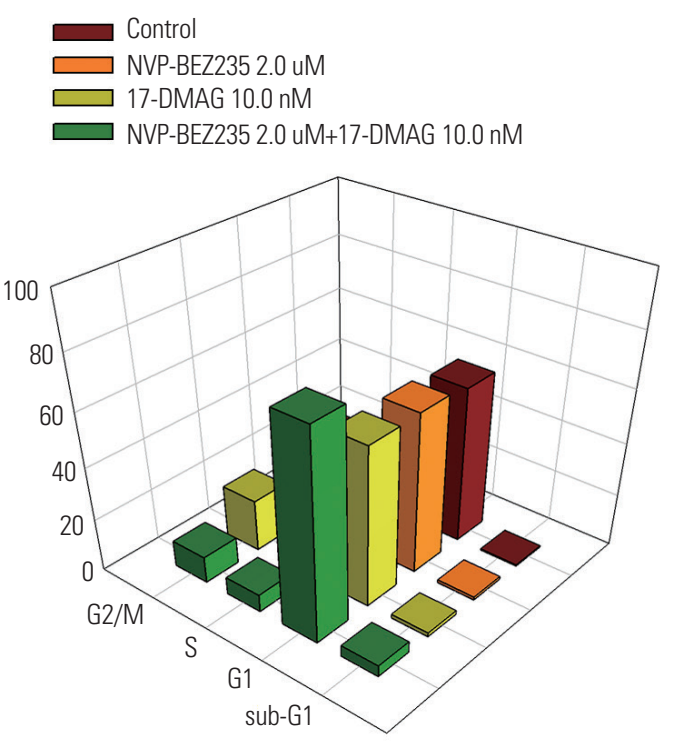

Fig. 6. Alterations in the cell cycle in T24R2 cells. T24R2 cells were treated with 17-DMAG (10 nM) alone or in combination with NVP-BEZ235 (2 $\mu M)$ for 48 hours, and changes in cell cycle were evaluated by flow cytometry. 

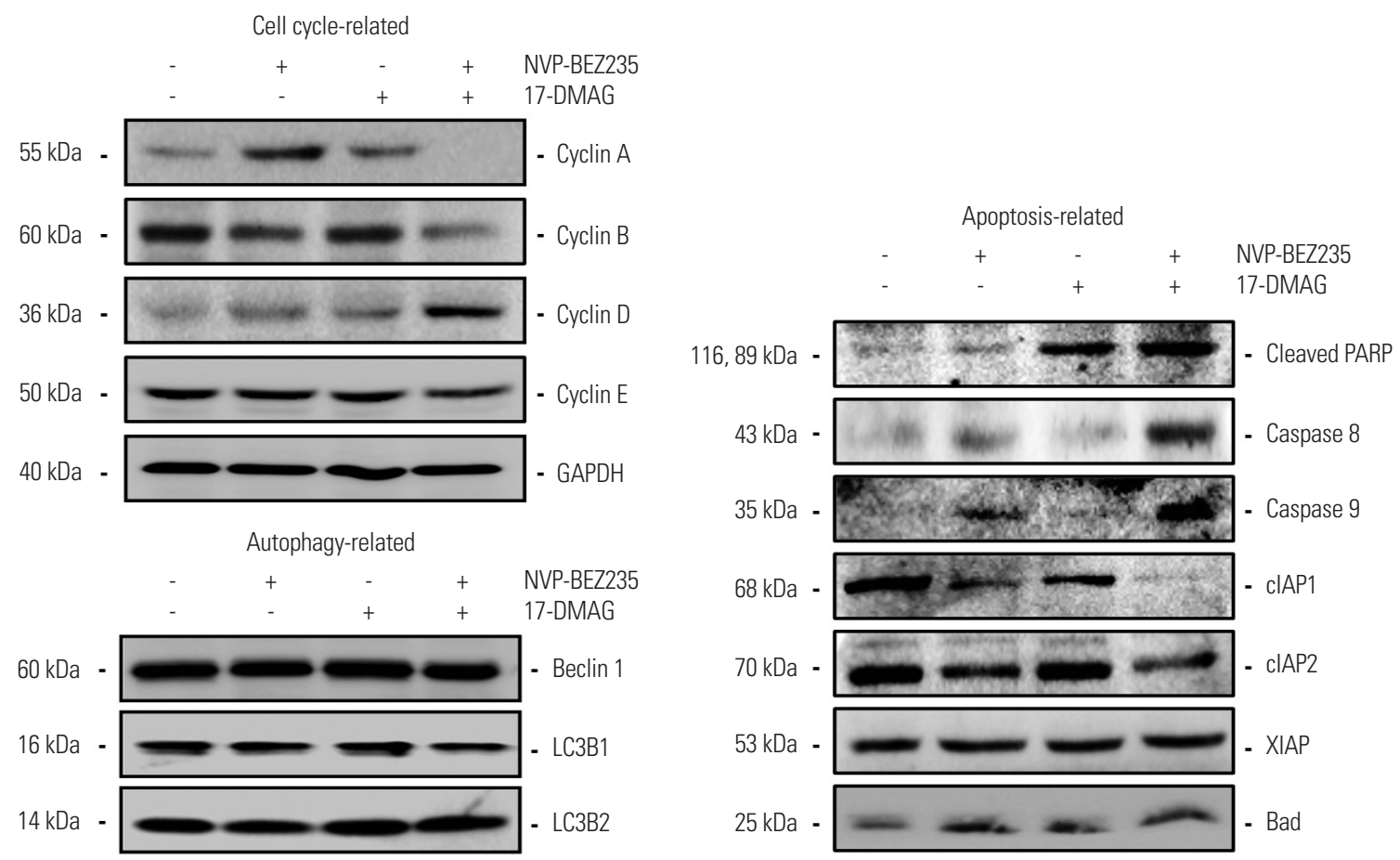

Fig. 7. Western blot analysis of proteins involved in apoptosis, cell cycle, and autophagy. T24R2 cells were treated with 17-DMAG (10 nM) alone or in combination with NVP-BEZ235 $(2 \mu \mathrm{M})$ for 48 hours, and expressions of relevant proteins were evaluated by Western blot analysis.

different types of cancer. HER2 belongs to the type 1 tyrosine kinase receptor family, which consists of four related receptors that form dimers with each other and is involved in cell cycle regulation and cell proliferation. Amplification of the HER2 gene and overexpression thereof at the protein level have been shown to be associated with oncogenesis. ${ }^{1,15}$ In a healthy environment, cells have low HER2 membrane protein content; however, but this is dramatically increased in cancer cells. Overexpression of HER2 protein has been reported in several malignancies, especially in gastric (20\%) and breast cancers (up to $30 \%$ ). ${ }^{16}$ In BCa, the expression frequency of HER2 has been reported to range from $25-100 \%$ in primary tumors and from $40-70 \%$ in corresponding metastases, which makes HER2 an attractive target for systemic therapy. ${ }^{1}$ Studies have shown that HER2 overexpression is associated with disease aggressiveness and more unsatisfactory outcomes, suggesting HER2 targeted therapy as a feasible option for locally advanced and metastatic HER2-amplified BCa. ${ }^{15,16}$

When HER2 proteins dimerize and bind to a ligand, the PI3K-Akt-mTOR and RAF-MEK-ERK pathways are activated. ${ }^{17}$ Signaling via the RAF-MEK-ERK cascade leads to phosphorylation of various proteins that have multiple cellular effects, mostly in cell cycle progression. ${ }^{18}$ Abnormal activation of the PI3K-Akt-mTOR signaling axis induces the development, progression, metastasis, and chemo-resistance in many tumors, including urothelial cancers. ${ }^{3}$ These findings have drawn attention to PI3K and mTOR signaling as promising antitumor targets. PI3K activation is believed to be involved in cisplatin resistance mechanisms through mutational activation of PI3K,
PTEN loss, and activation of alternative receptor tyrosine kinase. Such findings suggest the possible use of PI3K inhibitors in cisplatin-resistant cancers. ${ }^{6}$ Inhibition of mTOR has shown antitumor activity both in monotherapy and in combination with chemotherapy. In urothelial carcinoma cells, mTOR inhibition enhanced chemosensitivity by inducing cell cycle arrest at the sub-G1 and G1 growth phases and inhibiting VEGF production. The first generation mTOR inhibitors (e.g., rapamycin, temsirolimus, and everolimus) show synergistic activity with cisplatin, but yield inadequate treatment responses. ${ }^{5}$ These first generation mTOR inhibitors act by allosteric inhibition of mTORC1, but not mTORC2, which induces paradoxical activation of a survival axis by mTORC2-mediated Akt phosphorylation..$^{19,20}$ Therefore, the dual inhibition of PI3K/ mTOR or Akt/mTOR may overcome these feedback loops and, thus, prevent the development of resistance among cancer cells to targeted therapy. In support thereof, Moon, et al. ${ }^{3} \mathrm{dem}-$ onstrated a dose-dependent antitumor effect for the PI3K/ mTOR dual inhibitor NVP-BEZ235 in bladder cell lines. NVPBEZ235 blocked the negative feedback activation of Akt and showed a more significant antitumor effect, compared to the first generation rapalogues. However, NVP-BEZ235 monotherapy had an only partial inhibitory effect even at a relatively high dose $(0.5 \mu \mathrm{M}$ or higher $)$ and showed a transient antitumor effect lasting no more than 3 days. These findings were more pronounced in cisplatin-resistant T24R2 cells, compared to cisplatin-sensitive T24 cells, suggesting possible cross-resistance between NVP-BEZ235 and cisplatin in human BCa. NVPBEZ235 and cisplatin in combination showed significant syner- 


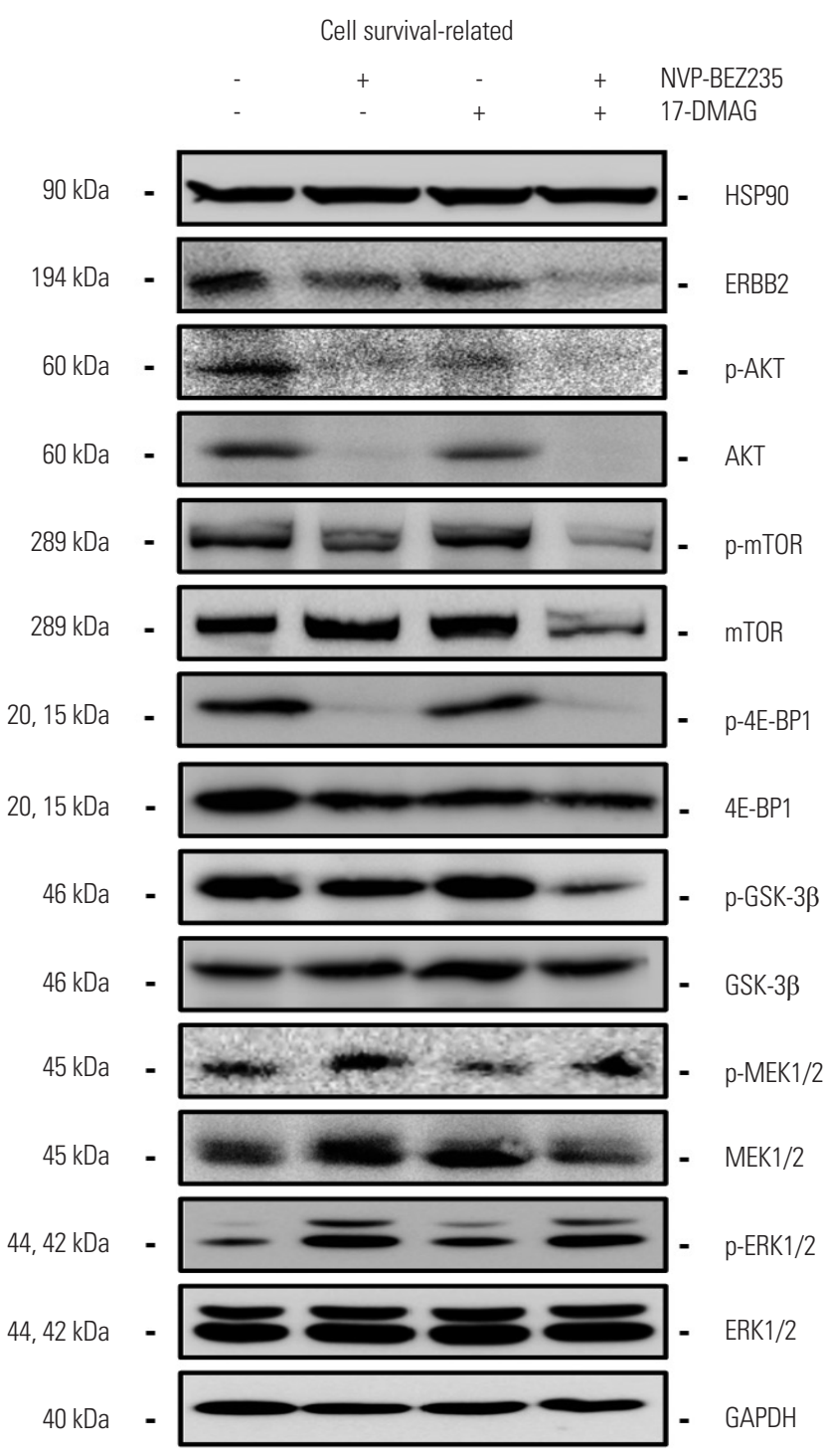

Fig. 8. Western blot analysis of proteins involved in cell survival. T24R2 cells were treated with 17-DMAG $(10 \mathrm{nM})$ alone or in combination with NVP-BEZ235 $(2 \mu \mathrm{M})$ for 48 hours, and changes in cell survival-related protein expression were explored using Western blot analysis.

gistic antitumor effects by eliciting cell cycle arrest and caspasedependent apoptosis in cisplatin-resistant bladder cancer cells, compared to treatment with either agent alone.

The activity of HER2 is affected and partly controlled by "molecular chaperones," which assure accurate folding of proteins, maintaining cellular integrity and homeostasis. In normal conditions, HSPs act as classical chaperones. Among several classes of HSPs, HSP90 is most abundant, and its loss of expression is related to poorer prognosis. ${ }^{7}$ Given that therapeutic resistance is attributed to diverse accumulating mutations in cancer cells, targeting multiple anti-apoptotic pathways is ideal. There are HSP90 client proteins other than Akt and ERK that act as vital components of various oncogenic pathways related to growth and survival, such as hypoxia-inducible factor-1a, erbB2, and inhibitor of $\kappa B$ kinase $\beta$, which mediates NF- $\kappa \mathrm{B}$ activation. ${ }^{21}$ Since these molecules are deeply involved in significant cancer pathways and play essential roles as oncogenes, the ubiquitous molecular chaperone HSP90 is a promising candidate thought which to enhance treatment responses. In several malignancies, such as gastric and breast cancer, HSP90 expression has been shown to be significantly associated with tumor aggressiveness and poor prognosis. Anti-HSP90 treatment for these cancers has shown potent benefits in numerous preclinical and clinical studies. ${ }^{9,12,15,17,22,23}$ In bladder cancer cells, 17-AAG clearly showed a dose-dependent and cell type-specific inhibition of cell proliferation, survival, and motility. ${ }^{24}$ Yoshida, et al. ${ }^{25}$ suggested that HSP90 inhibitors, even at a low concentration (17-DMAG or 17-AAG at $100 \mathrm{nM}$ ), would be potent enough to improve chemoradiation therapy responses by inactivating erbB2, Akt, and NF- $\mathrm{kB}$ without increasing toxicities. ${ }^{6}$ Tatokoro, et al. ${ }^{21}$ demonstrated that administration of HSP90 inhibitors at noncytocidal concentrations synergistically potentiated the cytotoxic effects of cisplatin on BCa-initiating cells in vitro and successfully sensitized cisplatin-resistant, BCa-initiating, cell-derived tumor xenografts to cisplatin. These data suggest that it may be feasible to utilize HSP90 inhibitors to improve the therapeutic outcomes of cisplatin-based combination chemotherapy for advanced BCa. ${ }^{21}$

Not only applying HSP90 inhibitor but combining it with other targeted therapies in conjunction with SOC may block compensatory signaling pathways and convey a clinical benefit. ${ }^{5}$ An optimal strategy for the combination is to target the same pathway vertically. ${ }^{26}$ Since Akt is one of the client proteins of HSP90, we hypothesized that a combined regimen with an HSP90 inhibitor (17-DMAG) and a PI3K/mTOR dual inhibitor (NVP-BEZ235) could augment the relatively low antitumor effects of each agent in cisplatin-resistant bladder cancer cells. In line with previous results, the antitumor effect of NVP-BEZ235 alone was self-limiting with an antitumor effect reaching a plateau at a concentration around $0.5 \mu \mathrm{M}$. However, the proliferation of both T24 and T24R2 cells were suppressed in a dose- and time-dependent manner when exposed to escalating doses of HSP90 inhibitor 17-DMAG. Synergy tests for the combination of NVP-BEZ235 and 17-DMAG with cisplatin in cisplatin-resistant bladder cancer cells also revealed significantly enhanced antitumor effects, compared to the effects of either drug alone. Fa-CI plots revealed that these two agents exert synergistic effects over a wide range of dose combinations in T24R2 cells. Dose reduction index analysis indicated that when this combination is applied to treat T24R2 cells, the $\mathrm{IC}_{50}$ of NVP-BEZ235 and 17-DMAG can be reduced by 1196.0- and 11.2- fold, respectively, indicating strong synergistic interactions within two drugs. 3D synergy test demonstrated in a synergy volume of $201.84 \mu \mathrm{M} / \mathrm{mL}^{2}$, with minimal antagonism, supporting the importance of this effect in vivo.

In human urinary bladder cancer cells, the diverse patterns of cell cycle arrest in response to 17-DMAG administration are associated with malignancy grade and cellular environment. 
Low malignancy p53wt cells, such as RT4, present with a prominent G1/S block, while p53 mutant T24 cells exhibit various patterns of cell cycle arrest. In a previous study, a combination of NVP-BEZ235 and cisplatin showed prominent cytostatic effects, rather than apoptogenic effects, as was confirmed by a mild increase in sub-G1 fractions, and elicited a marked increase in S phase fractions. In the present study, however, cotreatment of 17-DMAG and NVP-BEZ235 provided a slight increase in sub-G1 fractions and a marked increase in G1 phase cells in flow cytometry, suggesting prominent apoptotic effects. Western blot analysis demonstrated that the combined treatment resulted in a marked decrease in cyclin A and cyclin $\mathrm{B}$, while causing increased expression of cyclin D. These findings indicate that the antitumor mechanism of combined therapy is at least partly through G2/M phase cell cycle arrest. Moreover, combined treatment with 17-DMAG and NVP-BEZ235 suppressed the expression of anti-apoptotic proteins cIAP1 and cIAP2 and enhanced the cleavage of caspase $8 / 9$ and PARP, indicating induction of both extrinsic and intrinsic caspase-dependent apoptotic pathways. Akt, which is a crucial protein kinase of the complex cell signaling network and an essential member of the HSP90 complex, is downregulated upon 17-DMAG administration. The exposure of T24R2 cells to 17DMAG in combination with NVP-BEZ235 suppressed the phosphorylation of PI3K, mTOR, and its immediate targets GSK-3 $\beta$ and 4E-BP1. Simultaneous suppression of PI3K and mTOR with the deactivation of Akt and its downstream signaling reflected the antiproliferative and proapoptotic activities of 17DMAG and NVP-BEZ235 in combination with cisplatin in cisplatin-resistant bladder cancer cells. These results correspond with those reported by Chen, et al., ${ }^{6}$ wherein combination of a PI3K/mTOR dual inhibitor (NVP-BEZ235) and HSP90 inhibitor (NVP-AUY922) showed significant synergistic proapoptotic and antiproliferative effects in cholangiocellular carcinoma cells in vitro. Furthermore, they demonstrated that the HSP inhibitor NVP-AUY922 induced ER stress and mitochondrial damage fueled by oxidative stress when combined with NVPBEZ235. There were no apparent changes in the expression of the autophagy-related genes beclin 1, LC3B1, and LC3B2 in T24R2 cells after combined treatment with 17-DMAG and NVPBEZ235.

Previous studies have shown that 17-DMAG simultaneously inactivates both Akt and ERK signaling at non-cytocidal concentrations when combined with cisplatin in human bladder cancer cells. ${ }^{21}$ In our study, however, the combination of NVPBEZ235 and 17-DMAG decreased the expression of PI3K-related proteins (Akt, mTOR) while increasing the expression of MERK-ERK-associated proteins. Activation of MAPK/ERK pathway signaling is known as a resistance mechanism in mTOR inhibitor-based therapy. Roccaro, et al. ${ }^{27}$ demonstrated that NVP-BEZ235 upregulates ERK phosphorylation in Waldenstrom macroglobulinemia cells. NVP-BEZ235 monoand combination therapy with cisplatin in bladder cancer cell line upregulates MEK $1 / 2$, and ERK $1 / 2 .{ }^{3}$ Thus, we suspect that 17-DMAG did not reverse the effect of NVP-BEZ235 regarding ERK signaling. Such crosstalk indicates the MAPK/ERK pathway as another potential concomitant target through which to enhance the antitumor effects of combination therapy in cisplatin-resistant BCa.

The limitations of the current study include the in vitro study design and the small sample size. However, we were able to outline synergistic interactions between 17-DMAG and NVPBEZ235 in cisplatin-resistant BCa. Further comprehensive molecular studies, including in vivo studies, would be required to validate our findings and to further elucidate interactions between 17-DMAG and NVP-BEZ235 in cisplatin-resistant BCa.

In conclusion, combined treatment with the HSP90 inhibitor 17-DMAG and the PI3K/mTOR survival pathway inhibitor NVP-BEZ235 showed synergistic antitumor effects of eliciting cell cycle arrest at the G1 phase and induction of caspase-dependent apoptotic pathways in cisplatin-resistant bladder cancer cells.

\section{ACKNOWLEDGEMENTS}

This research was supported by Basic Science Research Program through the National Research Foundation of Korea (NRF) funded by the Ministry of Education (2012R1A1A2043 965).

\section{AUTHOR CONTRIBUTIONS}

Conceptualization: Young Deuk Choi and Hyung Joon Kim. Data curation: Cheol Yong Yoon, Hyung Joon Kim, and Mi Kyung Gong. Formal analysis: Cheol Yong Yoon and Mi Kyung Gong. Funding acquisition: Cheol Yong Yoon. Investigation: Hyung Joon Kim and Cheol Yong Yoon. Methodology: Cheol Yong Yoon, Hyung Joon Kim, and Mi Kyung Gong. Project administration: Hyung Joon Kim, Cheol Yong Yoon, and Mi Kyung Gong. Resources: Young Deuk Choi, Cheol Yong Yoon, and Hyung Joon Kim. Software: Young Deuk Choi and Cheol Yong Yoon. Supervision: Young Deuk Choi, Jaeku Kang, Mijin Yun, Nam Hoon Cho, and Sun Young Rha. Validation: Young Deuk Choi, Jaeku Kang, Mijin Yun, Nam Hoon Cho, and Sun Young Rha. Visualization: Hyung Joon Kim, Cheol Yong Yoon, and Mi Kyung Gong. Writing_original draft: Hyung Joon Kim. Writing_review \& editing: Hyung Joon Kim. Approval of final manuscript: all authors.

\section{ORCID iDs}

Hyung Joon Kim Mi Kyung Gong Cheol Yong Yoon Jaeku Kang Mijin Yun Nam Hoon Cho Sun Young Rha Young Deuk Choi https://orcid.org/0000-0003-3975-3045 https://orcid.org/0000-0002-9891-8711 https://orcid.org/0000-0002-2845-742X https://orcid.org/0000-0002-1712-163X https://orcid.org/0000-0002-0045-6441 https://orcid.org/0000-0002-2512-4531 https://orcid.org/0000-0002-8545-5797 https://orcid.org/0000-0002-8660-7940 


\section{REFERENCES}

1. Carlsson J, Wester K, De La Torre M, Malmström PU, Gårdmark T. EGFR-expression in primary urinary bladder cancer and corresponding metastases and the relation to HER2-expression. On the possibility to target these receptors with radionuclides. Radiol Oncol 2015;49:50-8.

2. Aragon-Ching JB, Trump DL. Systemic therapy in muscle-invasive and metastatic bladder cancer: current trends and future promises. Future Oncol 2016;12:2049-58.

3. Moon du G, Lee SE, Oh MM, Lee SC, Jeong SJ, Hong SK, et al. NVP-BEZ235, a dual PI3K/mTOR inhibitor synergistically potentiates the antitumor effects of cisplatin in bladder cancer cells. Int J Oncol 2014;45:1027-35.

4. Park BH, Lim JE, Jeon HG, Seo SI, Lee HM, Choi HY, et al. Curcumin potentiates antitumor activity of cisplatin in bladder cancer cell lines via ROS-mediated activation of ERK1/2. Oncotarget 2016;7:63870-86.

5. Chehab M, Caza T, Skotnicki K, Landas S, Bratslavsky G, Mollapour M, et al. Targeting Hsp90 in urothelial carcinoma. Oncotarget 2015;6:8454-73.

6. Chen MH, Chiang KC, Cheng CT, Huang SC, Chen YY, Chen TW, et al. Antitumor activity of the combination of an HSP90 inhibitor and a PI3K/mTOR dual inhibitor against cholangiocarcinoma. Oncotarget 2014;5:2372-89.

7. Lebret T, Watson RW, Molinié V, O'Neill A, Gabriel C, Fitzpatrick JM, et al. Heat shock proteins HSP27, HSP60, HSP70, and HSP90: expression in bladder carcinoma. Cancer 2003;98:970-7.

8. Lebret T, Watson RW, Molinié V, Poulain JE, O'Neill A, Fitzpatrick JM, et al. HSP90 expression: a new predictive factor for BCG response in stage Ta-T1 grade 3 bladder tumours. Eur Urol 2007;51: 161-6; discussion 166-7.

9. Lee JM, Lee SH, Hwang JW, Oh SJ, Kim B, Jung S, et al. Novel strategy for a bispecific antibody: induction of dual target internalization and degradation. Oncogene 2016;35:4437-46.

10. Citri A, Gan J, Mosesson Y, Vereb G, Szollosi J, Yarden Y. Hsp90 restrains ErbB-2/HER2 signalling by limiting heterodimer formation. EMBO Rep 2004;5:1165-70.

11. Neckers L. Hsp90 inhibitors as novel cancer chemotherapeutic agents. Trends Mol Med 2002;8:S55-61.

12. Kong A, Rea D, Ahmed S, Beck JT, López R, Biganzoli L, et al. Phase 1B/2 study of the HSP90 inhibitor AUY922 plus trastuzumab in metastatic HER2-positive breast cancer patients who have progressed on trastuzumab-based regimen. Oncotarget 2016;7: 37680-92.

13. Karkoulis PK, Stravopodis DJ, Konstantakou EG, Voutsinas GE. Targeted inhibition of heat shock protein 90 disrupts multiple oncogenic signaling pathways, thus inducing cell cycle arrest and programmed cell death in human urinary bladder cancer cell lines. Cancer Cell Int 2013;13:11.

14. Lee S, Yoon CY, Byun SS, Lee E, Lee SE. The role of c-FLIP in cispl- atin resistance of human bladder cancer cells. J Urol 2013;189: 2327-34

15. Soria F, Moschini M, Haitel A, Wirth GJ, Gust KM, Briganti A, et al. The effect of HER2 status on oncological outcomes of patients with invasive bladder cancer. Urol Oncol 2016;34:533.e1-10.

16. Nedjadi T, Al-Maghrabi J, Assidi M, Dallol A, Al-Kattabi H, Chaudhary A, et al. Prognostic value of HER2 status in bladder transitional cell carcinoma revealed by both IHC and BDISH techniques. BMC Cancer 2016;16:653.

17. Huang W, Wu QD, Zhang M, Kong YL, Cao PR, Zheng W, et al. Novel Hsp90 inhibitor FW-04-806 displays potent antitumor effects in HER2-positive breast cancer cells as a single agent or in combination with lapatinib. Cancer Lett 2015;356:862-71.

18. Knowles MA, Hurst CD. Molecular biology of bladder cancer: new insights into pathogenesis and clinical diversity. Nat Rev Cancer 2015;15:25-41.

19. Wangpaichitr M, Wu C, You M, Kuo MT, Feun L, Lampidis T, et al. Inhibition of mTOR restores cisplatin sensitivity through downregulation of growth and anti-apoptotic proteins. Eur J Pharmacol 2008;591:124-7.

20. Rao YK, Wu AT, Geethangili M, Huang MT, Chao WJ, Wu CH, et al. Identification of antrocin from Antrodia camphorata as a selective and novel class of small molecule inhibitor of Akt $/ \mathrm{mTOR}$ signaling in metastatic breast cancer MDA-MB-231 cells. Chem Res Toxicol 2011;24:238-45.

21. Tatokoro M, Koga F, Yoshida S, Kawakami S, Fujii Y, Neckers L, et al. Potential role of Hsp90 inhibitors in overcoming cisplatin resistance of bladder cancer-initiating cells. Int J Cancer 2012;131: 987-96.

22. Cao ZW, Zeng Q, Pei HJ, Ren LD, Bai HZ, Na RN. HSP90 expression and its association with wighteone metabolite response in HER2-positive breast cancer cells. Oncol Lett 2016;11:3719-22.

23. Slotta-Huspenina J, Becker KF, Feith M, Walch A, Langer R. Heat Shock Protein 90 (HSP90) and Her2 in Adenocarcinomas of the Esophagus. Cancers (Basel) 2014;6:1382-93.

24. Karkoulis PK, Stravopodis DJ, Margaritis LH, Voutsinas GE. 17-Allylamino-17-demethoxygeldanamycin induces downregulation of critical Hsp90 protein clients and results in cell cycle arrest and apoptosis of human urinary bladder cancer cells. BMC Cancer 2010;10:481.

25. Yoshida S, Koga F, Tatokoro M, Kawakami S, Fujii Y, Kumagai J, et al. Low-dose Hsp90 inhibitors tumor-selectively sensitize bladder cancer cells to chemoradiotherapy. Cell Cycle 2011;10:4291-9.

26. Yap TA, Omlin A, de Bono JS. Development of therapeutic combinations targeting major cancer signaling pathways. J Clin Oncol 2013;31:1592-605.

27. Roccaro AM, Sacco A, Husu EN, Pitsillides C, Vesole S, Azab AK, et al. Dual targeting of the PI3K/Akt/mTOR pathway as an antitumor strategy in Waldenstrom macroglobulinemia. Blood 2010;115: 559-69. 